Voix et Images

voixetimages

\title{
Bibliographie de Denise Desautels
}

\section{Patrick St-Amand}

Volume 26, numéro 2 (77), hiver 2001

Denise Desautels

URI : https://id.erudit.org/iderudit/201543ar

DOI : https://doi.org/10.7202/201543ar

Aller au sommaire du numéro

Éditeur(s)

Université du Québec à Montréal

ISSN

0318-9201 (imprimé)

1705-933X (numérique)

Découvrir la revue

Citer ce document

St-Amand, P. (2001). Bibliographie de Denise Desautels. Voix et Images, 26(2),

317-335. https://doi.org/10.7202/201543ar d'utilisation que vous pouvez consulter en ligne.

https://apropos.erudit.org/fr/usagers/politique-dutilisation/ 


\section{Bibliographie de Denise Desautels}

\section{Patrick St-Amand, Université du Québec à Montréal}

\section{Euvres}

\section{I.1. Poésie}

Comme miroirs en feuilles, avec dessin de Léon Bellefleur, Saint-Lambert, Éditions du Noroît, 1975, 90 p.

Marie, tout s'éteignait en moi, avec dessins de Léon Bellefleur, Saint-Lambert, Éditions du Noroit, 1977, 86 p.

La promeneuse et l'oiseau suivi de Journal de la promeneuse, avec gaufrure et dessin de Lucie Laporte, Saint-Lambert, Éditions du Noroît, 1980, 86 p.

En état d'urgence, avec dessin de Francine Simonin, Montréal, Éditions Estérel, 1982, $24 \mathrm{p}$.

L'écran, avec dessins de Francine Simonin, Saint-Lambert, Éditions du Noroît, 1983, $92 \mathrm{p}$.

: dimanche, Montréal, Éditions de La nouvelle barre du jour, 1985, 24 p.

Nous en reparlerons sans doute, en collaboration avec Anne-Marie Alonzo, à partir de cinq photographies de Raymonde April, Laval, Éditions Trois, 1986, s.p.

La répétition, avec photographies de La salle de classe, installation de Irene $\mathrm{F}$. Whittome, Montréal, Éditions de La nouvelle barre du jour, 1986, 64 p.

Écritures/Ratures, avec dessins de Francine Simonin, Saint-Lambert, Éditions du Noroît, coll. "Écritures/Ratures", 1986, s.p.

Un livre de Kafka à la main, avec photographies de Jocelyne Alloucherie, SaintLambert, Éditions du Noroît, 1987, 112 p.

Le signe discret, avec dessins de Francine Simonin, Lausanne, Éditions Pierre-Alain Pingoud, 1987, $48 \mathrm{p}$.

Mais la menace est une belle extravagance, avec photographies de Ariane Thézé, SaintLambert, Éditions du Noroit, 1989, 112 p.

Leģons de Venise, autour de trois sculptures de Michel Goulet, Saint-Lambert, Éditions du Noroît, 1990, s.p.

Le saut de l'ange, autour de photographies des ouvres de Martha Townsend, Montréal/ Le Noroît, Amay/L'Arbre à paroles, 1992, 96 p.

Cimetières: la rage muette, avec photographies de Monique Bertrand, Montréal, Éditions Dazibao, coll. "Des photographes ", 1995, 148 p.

"Ma joie ", crie-t-elle, avec dessins de Francine Simonin, Montréal, Éditions du Noroît, 1996, 106 p. 
Tombeau de Lou, autour de Visions domestiques, photographies d'Alain Laframboise, Montréal, Éditions du Noroît, 2000, 136 p.

\subsection{Récit}

Ce fauve, le Bonbeur, Montréal, l'Hexagone, 1998, 240 p.

\subsection{Correspondance}

Lettres'à Cassandre, en collaboration avec Anne-Marie Alonzo, postface de Louise Dupré, Laval, Éditions Trois, 1994, 128 p.

\subsection{Livres d'artiste}

Black Words, poésie, avec dessins originaux et impressions laser de Betty Goodwin, Paris, Éditions Collectif Génération, 1991, s.p.

Théâtre pourpre, poésie, avec peintures originales de Jean-Luc Herman, Paris, Éditions Jean-Luc Herman, 1993, s.p.

Le vif de l'étreinte, poésie, avec aquarelles originales de Claire Beaulieu, reliure de Jacques Fournier, Montréal, Éditions Roselin, 1994, s.p.

La passion du sens, poésie, livre-objet réalisé en collaboration avec Sylvia Safdie et Jacques Fournier, Montréal, Éditions Roselin, 1996, s.p.

L'acier le bleu, poésie, livre-objet et gravure de Jacqueline Ricard, Paris, Éditions Raina Lupa, 1996, s.p.

L'écho, la chambre, la nuit, poésie, triptyque dont chaque titre comprend deux gravures de Jacqueline Ricard, Paris, Éditions Raiña Lupa, 1996, s.p.

De la douceur, poésie, livre-objet réalisé en collaboration avec Jacqueline Ricard et Jacques Fournier, Montréal/Éditions Roselin, Paris/La Cour pavée, 1997, s.p.

Parfois les astres, poésie, livre-objet réalisé en collaboration avec Louise Dupré et Jacques Fournier, Montréal, Éditions Róselin, 2000, s.p.

\subsection{Documents audio}

Lettres à Cassandre, cassette-audio, réalisée en collaboration avec Anne-Marie Alonzo, Laval, Productions AMA, 1990.

Alternances, cassette-audio, réalisée en collaboration avec Hélène Dorion, poète, et Violaine Corradi, claviériste, Montréal, Éditions du Noroît/Production Angelo, 1992.

Autour du temps, anthologie de poètes québécois contemporains, cédérom, Montréal, Éditions du Noroît, 1997.

\subsection{Catalogues d'exposition}

Territoires d'artistes/Paysages verticaux, Québec, Éditions du Musée du Québec, 1989, $126 \mathrm{p}$.

Tombeau de René Payant, Montréal/Éditions du Centre d'exposition et de théorie de l'art contemporain, Laval/Éditions Trois, 1991, 132 p.

Peter Krausz, De natura (bumana), Montréal, Galerie d'art du Centre Saidye Bronfman, 1992, s.p. 


\subsection{Livres en collaboration}

Neuf textes, en collaboration avec Claude Beausoleil, Jean Yves Collette, Normand de Bellefeuille, Louise Dupré, Michel Gay, Marcel Labine, Line McMurray et Marie Claire Vaillancourt, Montréal, Éditions de La nouvelle barre du jour, 1985, 45 p.

Spirales, textes de Jean Leymarie et de Denise Desautels, avec photographies des collages de Catherine Hübschmann, ouvrage édité par Paola Gribaudo, Turin, Disegno/Diverso, 1994, s.p.

Ayot l'espiègle, Montréal, Département d'arts plastiques, Université du Québec à Montréal, 1996, s.p.

\section{I.8. Principaux textes radiophoniques}

Le cri, dramatique réalisée par Claude Godin et diffusée à Radio-Canada dans le cadre de l'émission La feuillaison, en 1982.

Les gitanes, dramatique réalisée par Claude Godin et diffusée à Radio-Canada dans le cadre de l'émission La feuillaison, en 1985.

La blessure, récit diffusé à Radio-Canada dans le cadre de l'émission Souvenirs d'enfance et de jeunesse, en 1986.

Voix, dramatique écrite à partir d'un tableau d'Umberto Boccioni, La strada entra nella casa. Enregistré à Cologne en 1986 dans une réalisation de Michel Corod, ce texte a été diffusé à Radio-Canada, Radio France, la Radio-Télévision belge de la Communauté française et la Radio suisse romande, en 1987.

Venise (variations sur l'utopie), dramatique réalisêe par Michel Corod et diffusée à Radio-Canada, Radio France et la Radio suisse romande, en 1989.

La voix de Martha, fiction poétique réalisée par Fernand Ouellette et diffusée à RadioCanada, en 1990.

La violoncelliste, dramatique réalisée par Claude Godin et diffusée à Radio-Canada, en 1990.

La répétition, texte poétique réalisé par Michel Corod (avec des illustrations scéniques de Peter Handke et de Thomas Bernhard) et diffusé à la Radio suisse romande, à l'automne 1993. (Ce texte a été sélectionné pour représenter la Radio suisse romande au Grand Prix Paul-Gilson de la Fiction radio 1994.)

\subsection{Préfaces, postfaces et commentaires}

"La mémoire souveraine $/$ "Memories Are Everything ", fiction en guise de préface, poésie de Pierre Nepveu, traduction de Judith Cowan, Ellipse, n' 32,1984 , p. 4046.

"Axes et eau de Louky Bersianik •, Arcade, n 10, 1985, p. 53-54.

-Droite et de profil de Anne-Marie Alonzon, Estuaire, $\mathrm{n}^{\circ}$ 10, 1986, p. 53-54.

"Issac, Agonie, Oubli ou Hagard (sur Les images de Louise Bouchard) ", Trois, vol. II, $\mathrm{n}^{\circ} 2,1986$, p. 49.

"Hommage à Germaine Guèvremont ", Le Bulletin, UNEQ, 31 mars 1994, p. 6-7.

"Deux ou trois remarques d'une poète québécoise sur Les prodiges ordinaires de André Schmitz", L'Arbre à paroles, Amay (Belgique), $\mathrm{n}^{\circ}$ 85, "André Schmitz", mai-juin 1995. 
"Seul le désir", postface de Geste de Anne-Marie Alonzo, Laval, Éditions Trois, 1997, p. 151-159.

Préface de Ma neige/Le signe du sable/Au même pôle, livrẹ d'artiste de Ève Cadieux, Éditions Ève Cadieux, 1998, s.p.

"Ce qui importe „, témoignage sur Ce qui importe, recueil de Hugues Corriveau, Lettres québécoises, $\mathrm{n}^{\circ} 93$, printemps 1999, p. 13.

"L'invitation au paysage", présentation de l'artiste Francine Larivée, Trois, vol. XV, $\mathrm{n}^{\text {os }} 1-2-3$, octobre 1999 , p. 119-120.

"Accompagnement ", note de lecture sur Le carré du ciel de Françoise Ascal, Gare maritime (Revue de la Maison de la poésie de Nantes et Région), numéro spécial "Françoise Ascal ", novembre 2000, p. 35-36.

\section{I.10. Participation à des anthologies}

Poètes québécois, sous la direction de Louise Blouin et Bernard Pozier, TroisRivières/Écrits des Forges, Sainte-Foy/Cégep de Sainte-Foy, 1987, p. 62-66.

Brise-lames/Antemurale (anthologie de la poésie moderne au Québec), sous la direction de Jean Yves Collette et Nicole Deschamps, Italie, Bulzoni Editore, 1990, p. 143-147.

La barre du jour/La nouvelle barre du jour, "Fin. (vingt-cinq ans de littérature, une anthologie), Montréal, Éditions de La nouvelle barre du jour, coll. «Première Lignen, 1990, p. 422-424 et 484.

Antbologie de la poésie des femmes au Québec, sous la direction de Nicole Brossard et Lisette Girouard, Montréal, Les Éditions du remue-ménage, 1991, p. 232-234.

Poètes québécois, sous la direction de Louise Blouin et Bernard Pozier, Trois-Rivières, Écrits des Forges/Koudhia, 1991, p. 44-45.

Le souffle du poème (anthologie de poètes du Noroît), sous la direction d'Hélène Dorion, Montréal, Éditions Pierre Tisseyre et Éditions du Noroît, 1993, p. 51-62.

Le Québec en poésie, sous la direction de Jean Royer, Paris, Gallimard, coll. a Folio junior en poésie ", 1995, p. 111.

Poètes québécois, sous la direction de Louise Blouin et Bernard Pozier, Paris, L'Orange bleue éditeur, 1996, p. 156-157.

«80 voix au féminin. (anthologie 1981-1996, préparée par Claudine Bertrand et Louise Cotnoir), Arcade, n $^{\text {os }} 35-36,1996$, p. 198-199.

Littérature québécoise, des origines à nos jours, sous la direction de Heinz Weinmann et Roger Chamberland, Montréal, Hurtubise HMH, 1996, p. 316.

Autour du temps (anthologie de poètes québécois contemporains), Montréal, Éditions du Noroît, 1997 , p. $85-88$.

Récital (33 voix de femmes), sous la direction de Christiane Laîfaoui et Jean-Claude Rossignol, Solignac (France), Éditions Le bruit des autres, 1998, p. 80-87.

Antologia della poesia contemporena del Québec, sous la direction de Titti Follieri, Milan, Crocetti Editore, 1998, p. 72-81.

"Poesie Quebeku *, sous la direction de Jana Boxberger, Tvar (Prague), n 12, 1998, p. 16-17.

Les dixièmes filles de Mnémosyne, sous la direction de Christiane Laïfaoui et JeanClaude Rossignol, Solignac (France), Éditions Le bruit des autres, 1999, p. 49-53. 
Les grands poèmes de la poésie québécoise, sous la direction de Joseph Bonenfant, Alain Horic et France Théoret, Montréal, l'Hexagone, 1999, p. 281-287.

Anthologie de la littérature québécoise, sous la direction de Michel Laurin, avec la collaboration de Michel Forest, $2^{e}$ édition, Montréal, Les Éditions CEC Inc., 2000, p. 256.

\section{I.11. Collaboration à des publications collectives, revues littéraires, journaux, etc. (Essais, réflexions sur l'écriture)}

"Pivoine, Fleur, Vivi, Miche, Lou et Nouche". (essai-fiction en réponse à Réginald Martel), La Presse, le samedi 24 janvier 1981, cahier C, p. 3.

"Le beau pétrin ", Liberté, n 134, "L'institution littéraire québécoise ", 1981, p. 128-129.

"Une lettre de tendresse ", "Le cahier spécial du Salon du Livre de Montréal ", Le Devoir, le samedi 28 novembre 1982.

-Who's Afraid of America?", Les cent lignes de notre américanité, avec photographies de Herménégilde Chiasson, Moncton, Éditions Perce-Neige, 1984, p. 73-76.

"Les cö̈ncidences du présent ", La nouvelle barre du jour, $\mathrm{n}^{\circ} 141$, "Vouloir la fiction ", septembre 1984 , p. 68-69.

"L'insoutenable vertige de la fiction ", *Avons-nous vécu 1984?", cahier spécial, Le Devoir, le samedi 4 novembre 1984.

"L'accent sur la fiction", Cabiers des arts visuels au Québec, n² 26, été 1985, p. 5.

"Vidéaste/Et pourquoi pas écrivaine? ", Écrits du Canada français, n 55, 1985, p. $105-$ 113.

"Poème de la durée/Texte ", Estuaire, n 37, "La séduction du romanesque ", 1985 , p. 25-29.

-La métamorphose des sirènes", L'agenda des femmes 1986, Montréal, Éditions du remue-ménage, 1986, s.p.

"L'énigme. Objets continus ", Choisir la poésie, Trois-Rivières, Les écrits des Forges, 1986, p. $30-33$.

- Roman (à propos de la question) •, Etc Montréal, no 7, "L'effritement des valeurs ", printemps 1989 , p. 34-35.

"Liminaire", La nouvelle barre du jour, $\mathrm{n}^{\text {os }}$ 218-219, "Femmes de lettres", mars 1989, p. 9.

"Deux ou trois choses que je sais d'elle", La solitude, sous la direction de Pierre Morency, Montréal, l'Hexagone, 1990, p. 39-44.

"Une archéologie de l'intime ", La littérature et la vie au collégial, sous la direction de Ernesto Sanchez, Montréal, Modulo Éditeur, 1991, p. 35-37.

- La fission de l'œuf. (essai-fiction), Liens interculturels. Solitude des autres, sous la direction de Norma Lopez-Therrien, Montréal, Éditions Logiques, 1992, p. 19-23.

"Des femmes dans la ville ", Arcade, n 25, "Ĺes urbaines ", automne 1992, p. 3.

"Le Livre de voûte/Le torrent d'Anne Hébert ", Le Sabord, $\mathrm{n}^{\circ}$ 35, automne 1993, p. 25 26. (Texte repris dans Art Le Sabord, $\mathrm{n}^{\circ}$ 52.) 
"L'œil au ralenti * (préface), L'Agenda 1984 (Revue $\mathrm{n}^{\circ} 4$ de l'Association des Artistes à la Bastille, Paris), septembre 1993, s.p.

«Du terrain vague à la tranchée" (essai-fiction), Mcbius, $\mathrm{n}^{\circ} 58$, "La résistance à l'écriture ", hiver 1993, p. 9-12.

"La voix de l'Autre ", Écrits du Canada français, n ${ }^{\circ} 80$, "Les livres qui nous ont faits", 1994, p. $105-115$.

"De l'écriture à la peinture, à la musique ou la mise en abyme de la voix ", L'écrivain et la musique, sous la direction de Louise Maheux-Forcier et Jean-Guy Pilon, Montréal, l'Hexagone, 1994, p. 81-87.

"Mais comment pourrait-il en être autrement?", Développement et rayonnement de la littérature québécoise: un défi pour l'an 2000, sous la direction de l'UNEQ, Montréal, Nuit blanche éditeur, 1994, p. 279-285.

"Vivre grande" (autoportrait), Lettres québécoises (dossier sur Denise Desautels), $\mathrm{n}^{\circ} 74$, été 1994, p. 6-7.

"Et dire la poésie?", Gare maritime (Revue de la Maison de la poésie de Nantes et Région), $\mathrm{n}^{\circ} 2$, mars 1995 , p. 23.

"Aimez-vous les oiseaux rapaces?", Trois, vol. X, no 3, "L'œuvre en collaboration", (Actes du colloque organisé par André Gervais et Paul Chanel Malenfant), printemps-été 1995, p. 93-106.

"Discours de réception à l'Académie des lettres du Québec ", Le Bulletin de l'Académie des lettres du Québec, $\mathrm{n}^{\circ}$ 16, décembre 1995, p. 24-28.

"L'une et l'autre, etc. ", Arcade, no 39, "Ces femmes qui nous inspirent", hiver 1997, p. 39-42.

"Lettre de Montréal ", Courant d'ombres (Paris), no 4, printemps 1997, p. 168-172.

*Trois questions, trois pièges ", Liberté, no 232, "Écrire l'amour, encore... *, août 1997, p. 47-53.

"Ce que je cherche à faire •, Estuaire, n 89 , „De l'écriture du poème*, novembre 1997, p. 71-79.

*La métamorphose" (essai-fiction), Voix singulières. Réflexion sur l'art.actuel des femmes, Montréal, La centrale/Les Éditions du remue-ménage, 1997, p. 59-65.

"Pourquoi écrivez-vous?*, propos recueillis par Sylvain Houde, Raymond Bertin, Nicolas Tittley, Marie Labrecque, Voir, semaine du 12 au 18 novembre 1998, p. 62.

"Liminaire ", Trois, vol. XV, ${ }^{\text {os }}$ 1-2-3, "Féminisme et création: Colloque des écrivains du 17 octobre 1998, octobre 1999, p. 7-8.

-Leurs livres du $20^{\mathrm{e}}$ siècle ", propos recueillis par Marie Labrecque, Raymond Bertin et Pascale Navarro, Voir, semaine du 11 au 17 novembre 1999, p. 42-43.

"De la première confidence ", Premiers mots de l'an 2000, sous la direction de Réjean Bonenfant et de Éric Laprade, Trois-Rivières, Éditions des Glanures, 2000 , p. 54.

"Tout en sachant que... ", Gare maritime (Revue de la Maison de la poésie de Nantes et Région), "Poésie contestataire des États-Unis ", mars 2000, p. 43.

"Qui vous a donné votre première chance?", sous la direction de Pascale Navarro, Voir, semaine du 13 au 19 avril 2000.

"Chère Françoise...", Arcade, no 49, "Lettres pour prendre l'ère ", printemps 2000, p. 37-39. 


\section{I.12. Collaboration à des publications collectives, revues littéraires, journaux, etc. (Poésie, fiction)}

"Poèmes ", Liberté, no 78, 1972, p. 42-44.

"La promeneuse et l'oiseau * (extrait), Estuaire, $\mathrm{n}^{\text {os }}$ 9-10, décembre 1978, p. 107-110.

"Du cri perçant ", La nouvelle barre du jour, $n^{\circ} 96$, novembre 1980, p. 11-14.

"Aires du temps ", La nouvelle barre du jour, n 105, septembre 1981, p. 9-10.

"Le chant de l'inouï", La nouvelle barre du jour, $\mathrm{n}^{\circ} 112$, "La complicité ", mars 1982, p. $81-89$.

"Le cours des événements" (fragment), La nouvelle barre du jour, $\mathrm{n}^{\text {os }}$ 122-123, "Écritures 83", février 1983, p. 90-95.

"En marge et en blanc/Carnet de voyage ", La nouvelle barre du jour, $\mathrm{n}^{\circ} 124$, "Femmes", mars 1983, p. 93-100.

"Des voix désirantes/Rumeurs", La nouvelle barre du jour, $\mathrm{n}^{\text {os }} 127-128$, "Ce qui les séduit n, juin 1983, p. 75-82.

.Oui elle rognerait le temps...", Lèvres urbaines, $\mathrm{n}^{\circ} 1,1983, \mathrm{p} .13$. (Ce texte a été repris dans VWA, revue littéraire suisse, hiver 1984-1985, "La nouvelle poésie au Québec n, textes choisis et présentés par Claude Beausoleil.)

"Elle aurait aimé le bon moment... ", Poetry Agenda Poésie 1984, Montréal, La Compagnie des muses, 1984, s.p.

"Le bateau rouge" (texte écrit à partir de Red Wood Boat on Stand de Sylvain P. Cousineau), Montréal Now!, $\mathrm{n}^{\circ}$ 1, 1984, p. 7.

"Via Moncton/Le texte amoureux", Les cent lignes de notre américanité, avec photographies de Herménégilde Chiasson, Moncton, Éditions Perce-Neige, 1984, p. 127-130.

"La voix de Laurie ", La nouvelle barre du jour, n 138, avril 1984, p. 5-15.

"La mise en mots des silences rouges ", Estuaire, $\mathrm{n}^{\circ} 31$, printemps 1984, p. 21-30.

"Maquillée ", La nouvelle barre du jour, n 140, "Sortie/Exit ", juin 1984, p. 55-56.

"L'aveu/13 juillet/13h13 ", La nouvelle barre du jour, n 143, "Lire autrement ", 1984, p. $51-59$.

"Note/Éphémère ", Estuaire, nos 32-33, *Poésie 84 ", été-automne 1984, p. 53-55.

"Jardin de ville/Performance ", La nouvelle barre du jour, n ${ }^{\circ} 150$, "Party mixte *, 1985, p. 51-58.

"Été 1984/Les premier, deuxième et troisième jours ", Dalbousié French Studies, "La poésie québécoise depuis 1975 ", sous la direction de Eva Kushner et Michael Bishop, 1985, p. 105-107.

"Nous en reparlerons sans doute", en collaboration avec Anne-Marie Alonzo, à partir d'une photographie de Raymonde April, La nouvelle barre du jour, $\mathrm{n}^{\circ} 163$, "Double signature ", 1985, p. 35-39.

“Chants ", Passages, nº " "D'ici et d'ailleurs ", hiver 1986, p. 27-31.

"Là ", Trois, vol. I, no 3, printemps 1986, p. 48.

"Écritures/Ratures" (extraits), Estuaire, n ${ }^{\text {os }}$ 40-41, „L'Art poétique ", 1986, p. 60-62. 
"L'archéologue *, Canadian Fiction Magazine, numéro spécial sur la fiction théorique organisé par Tessera, $n^{\circ} 57,1986$, p. 107-116. (La traduction anglaise, "The Archeologist ", est de Suzanne de Lotbinière-Harwood.)

"Trois murs ", La nouvelle barre du jour, $\mathrm{n}^{\circ} 184$, "La littérature et après ", octobre 1986, p. 5-15.

"Enfance. L'éternelle conséquence ", Urgences, n 15, "Épigraphiques *, octobre 1986, p. 9.

"Sans titre. (Installation de mots et d'images réalisée en collaboration avec Monique Régimbald-Zeiber), La nouvelle barre du jour, $\mathrm{n}^{\text {os }} 189-190$, "Installations/Fictions ", décembre 1986, s.p.

"La répétition. (extrait), Vagabondages (France), no 66, "Poésie québécoise ", janvier/ février/mars 1987, p. 39.

"Le signe discret " (extrait), Le Journal des poètes (Belgique), n 3, mai 1987.

"Lettre à Cassandre ", La Parole métèque, no 3, "Dossier Anne-Marie Alonzon, automne 1987, p. 9.

"La mémoire amnésique •, Estuaire, n 45, "Le plus mauvais poème au monde", été 1987, p. 9-10.

*Un film de Godard. (sous le pseudonyme d'Alice Brousseau), Urgences, $\mathrm{n}^{\circ} 20$, "Appellation contrôlée", mai 1988, p. 11-12.

"Essai de voix ", Arcade, n 15, "Paysages intérieurs ", février 1988, p. 15-18.

"Le triangle , Trois, vol. III, no 3, printemps/été 1988, p. 110-111.

"Utopie ", Estuaire, n 50 , "Poésie 88 ", automne 1988, p. 37-38.

"La blessure ", Enfances et jeunesses, Montréal, Les entreprises Radio-Canada, 1988, p. 105-112.

"Dans une ville étrangère ", Montréal des écrivains, sous la direction de Louise Dupré, Bruno Roy et France Théoret, Montréal, UNEQ/l'Hexagone, 1988, p. 75-78.

"Tableaux", Estuaire, n 52, "Dans la vie des choses", printemps 1989, p. 19-26.

"Fait(s) de mémoire ", Trois, vol. IV, no 4, hiver 1989, p. 13-17.

"Lettres ouvertes ", La nouvelle barre du jour, $\mathrm{n}^{\text {os }}$ 218-219, .Femmes de lettres", mars 1989, p. 97-110.

"Une histoire future ", La nouvelle barre du jour, nos 220-221, "Autoportraits ", avril 1989, p. 137-143.

"Au restaurant ", $X Y Z$. La revue de la nouvelle, $\mathrm{n}^{\circ}$ 18, "La vérité ", mai 1989, p. 4-7.

"Mais la menace est une belle extravagance. (extrait), Nouveaux Cabiers méditerranéens, $\mathrm{n}^{\text {os }} 151-152$, "Souffles", automne 1989, s.p.

"Denise Desautels et Robyn Sarah", choix de textes, traduction de Ellipse, n 43, 1990, p. 18-51.

"Sans titre ", Estuaire, n 58, "La rumeur des lieux ", décembre 1990, p. 5-8.

"La voix de Martha ", Arcade, $\mathrm{n}^{\circ}$ 21, "Bruits et silences ", printemps 1991, p. 12-16.

"Le saut de l'ange" (extrait), Urgences, $n^{\circ} 33$, "Poésies parallèles: France-Québec ", octobre 1991, p. 47-50. 
"Sous les débris, l'ange *, Le Verbe et l'image (Revue $\mathrm{n}^{\circ} 2$ de l'Association des Artistes à la Bastille, Paris), automne 1991, p. 28.

"Les âmes voyageuses", Estuaire, nos 65-66, "Montréal ", automne 1992, p. 28-30.

"Sur les balcons fleuris ", Arcade, no 25, "Les urbaines", automne 1992, p. 37-40.

"Et l'homme... ", Trois, vol. VIII, n 3, printemps-été 1993, p. 189.

" La couleur se resserre •, Estuaire, no 70, automne 1993, p. 43-50.

"Le visage de la couleur "L'agenda 1984 (Revue $\mathrm{n}^{\circ} 4$ de l'Association des Artistes à la Bastille, Paris), septembre 1993, s.p.

"Le saut de l'ange. (extraits traduits par Pascale Agnew et Ciaran O'Driscoll), Cyphers (Dublin), no 38, hiver 1993, p. 41.

"Traductions croisées", La Traductière (Paris), no 11, "Les yeux du siècle", juin 1993, p. $10-13$ et p. $19,21,23,47,48,53$.

"Vues de près", West Coast Line (Vancouver), n 12, hiver 1993-1994, p. 56-58.

"La cuve de Vincent " (extrait), Le Sabord, n 36, "Objet Lime", hiver 1994, p. 9.

"Poèmes choisis", La Porte des poètes (Paris), no 26, "Poésie au féminin ", novembre 1993/mars 1994, p. 8.

"Le saut de l'ange" (extraits), La Porte des poètes (Paris), n 24, "Prose et poésie ", septembre-novembre 1994, p. 57.

" "Ma joie", crie-t-elle "(extrait), Courant d'ombres (Paris), no 1, printemps 1995.

" "Ma joie", crie-t-elle " (extrait), Estuaire, nos 80-81, mars 1996, p. 81.

"Cimetières: la rage muette" (extraits), Le Moule à gaufre (Paris), no 14, "Québec francophonie ", hiver 1996, p. 147-165.

"Arbre et os ", Bacchanales (Grenoble), numéro spécial "L’arbre ", juin 1996.

"Langue-mémoires ", Ressac, sous la direction de René Derouin, Montréal, l'Hexagone, 1996, p. 104 .

"La Polonaise ", Mcebius, nos 69-70, "La mémoire ", automne 1996, p. 193-199.

"L'autre père", Les Écrits, n 88 , automne 1996, p. 31-37.

"L'œil était sur le t-shirt et me regardait ", Trois, vol. XI, n 3, automne 1996, p. 162.

"Le fiancé. (Ce fauve, le Bonbeur, extrait), XYZ. La revue de la nouvelle, $\mathrm{n}^{\circ} 48$, "Taches", hiver 1997, p. 32-35.

"Le frère ", Arcade, no 39, "Ces femmes qui nous inspirent ", hiver 1997, p. 43-44.

" "Ma joie", crie-t-elle (extrait), Arcade, n 40, "Voix internationales", printemps 1997, p. 55-57.

"L'Étrangère ", Art Le Sabord, n 47, automne 1997, p. 8-10.

"Les grandes filles ", Mobius, no 78, “S'écrire jeune ", automne 1998, p. 80-84.

"L'orpheline imaginaire ", Les Écrits, no 93 , automne 1998, p. 5-12.

"C'est pour regarder la vie...", Estuaire, no 93, "Le vierge exquis", septembre 1998, p. 47-50.

"Cimetière : la rage muette. (extraits), Les Cabiers de poésies-rencontres (Lyon), $\mathrm{n}^{\text {os }} 44-$ 45, janvier 1999, p. 93-108. 
"C'est pour regarder la vie ", Europe (Paris), no 839, "Cahier de création ", mars 1999, p. $217-219$.

"Tu vieillis de plus en plus vite, et je te suis de près" (extrait), Lieux d'être (France), $\mathrm{n}^{\circ} 28$, "Québec: lieux d'échange ", été 1999, p. 44-46.

"Comme dans un tableau de Magritte", Estuaire, n० 98, septembre 1999, p. 38-43.

"Ultime cajolerie, ultime douceur", Midi (Paris), n 8, octobre 1999, p. 20-21.

"La danseuse", Trois, vol. XIV, $\mathrm{n}^{\text {os }} 2-3,1999$, p. 229.

"La poésie, l'espoir ", Estuaire, $\mathrm{n}^{\circ} 99$, "Le dernier poème du millénaire ", novembre 1999, p. 63.

"Une grande pièce blanche, presque vide ", Liberté, $\mathrm{n}^{\circ} 246$, "La chambre des poètes ", décembre 1999, p. 29-33.

"Tombeau de Lou" (extrait), Estuaire, no 101, mai 2000, p. 9-14.

"La libraire ", La maison du rêve, fictions, sous la direction de Simone Sauren, Montréal, l'Hexagone, 2000, p. 55-60.

\section{I.13. Collaborations diverses avec des artistes}

Texte intégré à des œuvres de Monique Régimbald-Zeiber, reproduites dans le numéro "Installations/Fictions" de La nouvelle barre du jour et exposées à la galerie Graff en décembre 1986.

Poèmes pour le dépliant qui accompagne l'exposition de Dominique Blain, Eva Brandl et Sylvie Blocher, et produit par la Galerie Christiane Chassay de Montréal, en 1990.

"De l'inquiétude ", texte intégré à un livre, FIGURATION transFIGURATION, regroupant les ouvres d'une quinzaine d'artistes et de trois écrivains, sous la direction de Pierre Ayot (Montréal, Galerie Graff, 1990).

Poème (traduction de Suzanne de Lotbinière-Harwood) pour Island de Martha Townsend, publié par Artspeak Gallery, Vancouver, 1991.

Participation à une œuvre collective (Gail Bourgeois, Suzanne Girard, Suzanne Paquet, Denise Desautels, Claire Paquet), Différences et sites (Montréal, La Centrale, 1992).

" "Voir", tu as dit "voir" ", placard "poésie-peinture ", sérigraphie de Jean-Luc Herman, Paris, 1993.

Poèmes (repris dans "Ma joie", crie-t-elle) intégrés à Sente, une chorégraphie de Lucie Grégoire pour le spectacle "Corps et écriture * organisé par l'UNEQ. Agora de la danse, Montréal, 1994. Spectacle repris, entre autres, à Station Danse, dans le métro Berri-UQÀM, en avril 1996. Spectacle revu et augmenté, avec la collaboration du musicien Rainer Wiens, présenté à la galerie L'œil de poisson, à Québec, en février 1998.

Texte pour le carton d'invitation à l'exposition des cuvres de Sylvia Safdie, présentée par Joyce Yahouda Art contemporain, du 19 novembre au 10 décembre 1994. ("So many precious objects... $n$, traduction de Judith Cowan.)

Hommage à Pierre Ayot, lu à la Galerie Graff, le 7 mai 1995, et repris dans Le Devoir du 10 mai 1995.

Poèmes intégrés à des ceuvres de Sylvia Safdie, présentées au Centre d'Exposition Circa, du 6 mai au 17 juin 1995, dans le cadre de l'exposition intitulée Écrits pour voir. 
Tampon d'artistes, réalisé en collaboration avec Michel Goulet, dans le cadre du Festival de la littérature 1997, édité par le Centre international d'art contemporain de Montréal et l'Union des écrivains et des écrivaines du Québec.

Poème pour le dépliant qui accompagne l'exposition La cloche de Linda Covit, présentée au Musée de la ville de Lachine du 17 août au 12 octobre 1997.

Extraits de Cimetières: la rage muette, intégrés à Figures du temps, une pièce de musique électroacoustique de Monique Jean, présentée aựThéâtre de la Chapelle, en février 1999.

Poème intégré à l'œuvre de Cozic, La règle de trois, pour l'exposition Matières des mots, présentée à la galerie Evelyne Guichard à La Côte-Saint-André, en novembre 1998, à la Vénerie à Bruxelles, en janvier 1999, et au Centre d'Exposition Circa de Montréal, en novembre 1999; repris dans le catalogue qui accompagne l'exposition.

Textes poétiques (un inédit et trois extraits de Leçons de Venise) pour le dépliant Nef pour quatorze reines, œuvre de Rose-Marie Goulet à la mémoire des femmes de Polytechnique, Service de la culture de la ville de Montréal, décembre 1999.

Poème qui encercle Sonores et nus, une eau-forte de Françoise Sullivan (Montréal, Éditions Lucie Grégoire danse, 2000).

Poème intégré à l'installation de Michel Goulet, Porter le mur comme le masque, présentée à la galerie Occurrence, en avril 1999, et au Centre culturel canadien à Paris, du 15 juin au 15 septembre 2000. Repris dans le catalogue Porter le mur comme le masque, Montréal, Les 400 coups, 2000.

\section{l.14. Direction de numéros spéciaux}

La nouvelle barre du jour, $\mathrm{n}^{\circ} 124$, "Femmes", en collaboration avec Carole David et Renée-Berthe Drapeau, mars 1983, $110 \mathrm{p}$.

La nouvelle barre du jour, $\mathrm{n}^{\text {os }} 189-190$, "Installations/Fictions", en collaboration avec Gilles Daigneault, 1986, s.p.

La nouvelle barre du jour, $\mathrm{n}^{\text {os }}$ 218-219, "Femmes de lettres ", avril 1989, 116 p.

Arcade, $\mathrm{n}^{\circ}$ 25, "Les urbaines", automne 1992, p. 3-47.

Trois, vol. XV, $\mathrm{n}^{\text {os }} 1-2-3$, "Féminisme et création: Colloque des écrivains du 17 octobre 1998 ", octobre 1999, p. 5-115.

\section{Prix et distinctions}

Le texte dramatique Voix a été primé par les radios publiques de langue française, en 1987.

Grand prix de la poésie du Journal de Montréal, en 1990, pour Mais la menace est une belle extravagance.

Grand prix de la poésie de la Fondation Les Forges, en 1991, pour Leçons de Venise.

Prix de la poésie du Gouverneur général du Canada, en 1993, pour Le saut de l'ange.

Prix du Signet d'or en poésie de Radio-Québec, en 1993, pour Le saut de l'ange.

Prix Terrasses Saint-Sulpice de la revue Estuaire, en 1993, pour Le saut de l'ange.

Élection à l'Académie des lettres du Québec en 1995. 
Médaille (échelon vermeil) de la ville de Paris en 1999.

Grand prix de poésie de la société Radio-Canada, en 1998, pour "Ma sisyphe" (trois extraits de Tombeau de Lou).

\section{Réception critique et études}

\section{III.1. Articles de fond, chapitres de livres, etc.}

ARCAND, Pierre-André, "Le Noroît", Livres et auteurs québécois 1975, Sainte-Foy, Presses de l'Université Laval, 1976, p. 122-124.

BORDELEAU, Francine, "L'écriture au féminin existe-t-elle? n, Lettres québécoises, n 92 , hiver 1998, p 14-18.

BROCHU, André, Tableau du poème. La poésie québécoise des années quatre-vingt, Montréal, XYZ, coll. "Documents ", 1994, p. 155-158.

BROSSARD, Nicole, "Texte de présentation de Denise Desautels à l'Académie des lettres du Québec ", Le Bulletin de l'Académie des lettres du Québec, $\mathrm{n}^{\circ}$ 16, décembre 1995, p. 21-24.

CALLE-GRUBER, Mireille, "Le double registre ou le moment spectral de l'œuvre d'art ", Trois, vol. X, n 3, "L'œuvre en collaboration", printemps-été 1995, p. 21-29.

CHAMBERLAND, Roger, "Veilleur, qu'en est-il de la nuit? „, Découvrir le Québec (Guide culturel), Sainte-Foy, Les Publications Québec français, 1987, p. 52-56.

CLOUTIER, Guy, "Le dehors et le dedans", Entrées en matière(s), Montréal, l'Hexagone, 1988, p. 80-82.

CORRIVEAU, Hugues, "Le saut de la promeneuse ", Lettres québécoises, n 74, été 1994, p. 9-11.

CORRIVEAU, Hugues, "La poésie québécoise à l'étranger", Salon du livre de Paris, Montréal, Comité d'éditeurs pour les Salons et Foires à l'étranger, 1994, p. 9-11.

DE BELLEFEUILLE, Normand, "Mon nom est légion", Trois, vol. $\mathrm{X}, \mathrm{n}^{\circ} 3$, "L'œuvre en collaboration ", printemps-été 1995, p. 121-135.

DUPRÉ, Louise, "Poetry Returns to Love", traduction en anglais d'Arlette Francière, Ellipse, $\mathrm{n}^{\circ} 39,1988$, p. 11-19. Repris en français sous le titre "Quand la nouvelle poésie devient amoureuse ", Trois, vol. IV, hiver 1989, p. 19-24.

DUPRÉ, Louise, "Denise Desautels: la pensée du poème ", Études françaises, vol. XXIX, $\mathrm{n}^{\circ} 3$, hiver 1994, p. 41-50.

ESCOMEL, Gloria, "Pourquoi écrire aujourd'hui? ", La Vie en rose, décembre/janvier 1985 , p. $54-55$.

GAUDET, Gérald, "La passion et ses doutes", La passion mélancolique, Estuaire, n 95 , 1998 , p. $57-63$.

GERVAIS, André, "Bibliographie des œuvres en collaboration", Trois, vol. X, $\mathrm{n}^{\circ} 3$, "L'œuvre en collaboration", printemps-été 1995, p. 11-20.

GOULET, Michel, "Frapper aux portes et abattre les murs ", Trois, vol. X, n 3, "L'œuvre en collaboration ", príntemps-été 1995, p. 71-92.

LAMARCHE, Lise, "Regards obliques dans les galeries souterraines ", Trois, vol. XI, $\mathrm{n}^{\circ} 1$, 1995, p. 1-10. 
LAMARRE, André, "Complicité et duplicité. Deux pratiques de l'écrit d'art", Trois, vol. $\mathrm{X}, \mathrm{n}^{\circ}$ 3, "L'œuvre en collaboration ", printemps-été 1995, p. 137-146.

NEPVEU, Pierre, "A Woman with no Special Qualities" (préface), Ellipse, n 43, 1990, p. 9-15. (Ce numéro était consacré aux œuvres de Denise Desautels et de Robyn Sarah, avec extraits en traduction.)

OUELLET, Pierre, "Voir double. Réécritures du regard", Trois, vol. X, $\mathrm{n}^{\circ} 3$, "L'œuvre en collaboration ", printemps-été 1995, p. 147-164. (Repris dans Ombres convives, Montréal, Éditions du Norôt, 1997, p. 121-140.)

PARÉ, François, "Me voici, c'est moi, la femme qui pleure ", Les littératures de l'exiguitté, Hearst (Ontario), Éditions Le Nordir, 1992, p. 91.

\section{III.2. Entretiens et portraits d'auteure}

CAMPION, Blandine, "Archéologue de l'intime ", Le Devoir, 13 mars 1999, p. E-21.

CAMPION, Blandine, "La demeurance", Spirale, no 168, septembre-octobre 1999, p. 16-17.

CHOUINARD, Marie-Andrée, "Archéologue de l'intime ", Le Devoir, 26 septembre 1998, p. D-1.

FOLLIERI, Titti, "Otto poeti contemporanei del Québec", Poesia (Milan), n 112, décembre 1997, p. 47-61 (présentation de poètes québécois et traduction d'extraits).

GALLON, Marie-Lise, "Oiseau rapace et fictionnaire autobiographique ", Gare maritime (Revue de la Maison de la Poésie de Nantes et Région), n 2, mars 1995, p. 17-21.

KÉCHICHIAN, Patrick, "Lyre au Québec", Le Monde, cahier du Salon du livre, 19 mars 1999, p. 5.

LAMARRE, André, "Denise Desautels et Francine Simonin", Le Sabord, $\mathrm{n}^{\circ} 36$, hiver 1994, p. 24-27.

MOLIN VASSEUR, Annie, "J'hésite entre le deuil et l'étèrnité: entretien avec Denise Desautels ", Arcade, no 31, "La dolce vita ", automne 1994, p. 53-65.

NAVARRO, Pascale, :Image de marque ", Voir, semaine du 7 au 13 mars 1996, p. 31.

NAVARRO, Pascale, "Vingt écrivains qui comptent ", Livres Hebdo (Paris), no 324, 1999, p. 84-86.

NICOLINI, Élisabeth, "Le feu sous la glace", La Vie (Paris), semaine du 18 au 24 mars 1999 , p. 52-55.

\section{III.3. Comptes rendus et critlques de livres, documents audio et autres}

\section{III.3.1. Comme miroirs en feuilles}

BEAUSOLEIL, Claude, "Un vent de Noroît ", Hobo-Québec, nº 25, automne 1975.

DIONNE, René, “Poésie •, University of Toronto Quaterly, vol. XLV, n 4, été 1976, p. 346-353.

DOSTIE, Gaétan, *Quand la substance flanche et que le désastre s'étend sans limites ", Le Jour, 4 octobre 1975, p. 15.

LARUE-LANGLOIS, Jacques, "Une poésie nommée amour •, Le Livre d'ici, n 11, 1975.

NEPVEU, Pierre, "Les nouvelles voix en poésie: les vaches maigres", Lettres québécoises, $\mathrm{n}^{\circ} 2$, mai 1976 , p. 12-14. 
PAGEAU, René, "Les premiers chants de Denise Desautels", L'Information médicale, 2 mars 1976 , p. 32.

\section{III.3.2. Marie, tout s'éteignait en moi}

ANONYME, "Marie, tout s'éteignait en moi ", Le Quotidien, 25 février 1978, p. C-4.

DELISLE, Claude, "Marie, tout s'éteignait en moi", Livres et auteurs québécois 1977: revue critique de l'année littéraire, Sainte-Foy, Les Presses de l'Université Laval, 1978, p. 156-157.

HAMEL, Marie-Andrée, "L'odyssée d'une femme ", Le Livre d'ici, vol. III, n 51, 1978.

NOLIN, Jacques, "Marie, tout s'éteignait en moi ", Nos livres, novembre 1978, p. 372.

PARADIS, Suzanne, "La poésie du "cercle": lumière ou silence ", Le Devoir, 6 mai 1978, p. 35.

\section{III.3.3. La promeneuse et l'oiseau}

BAYARD, Caroline, "Poésie", Letters in Canada, été 1981, p. 46.

BEAULIEU, Michel, "Denise Desautels, Oui... Une délivrance ", Le Livre d'ici, 17 septembre 1980.

BELLEMARE, Madeleine, "La promeneuse et l'oiseau", Nos livres, vol. 11, octobre 1980, p. 293.

CORRIVEAU, Hugues, "Des voix pluriellés ", Spirale, n 12, octobre 1980, p. 12.

CÔTÉ, Claire, "La promeneuse et l'oiseau", Le Bulletin Pantoute, $\mathrm{n}^{\circ} 4$, décembre/ janvier/février 1981, p. 5.

COTNOIR, Louise, "Sans titre ", Livres et auteurs québécois 1980, Sainte-Foy, Les Presses de l'Université Laval, 1981, p. 104-105.

CRÉPEAU, Jean-François, "Lire de la poésie l'automne ", Le Canada français, 29 octobre 1980 , p. C-6.

GIGUÈRE, Richard, "L'emploi d'écrire ", Lettres québécoises, nº 19, automne 1980, p. 32 35.

HAECK, Philippe, "Une voix amie ". La nouvelle barre du jour, $\mathrm{n}^{\circ}$ 99, février 1981, p. 63-64. (Repris dans La table d'écriture: poétique et modernité, Montréal, VLB éditeur, 1984, p. 149-151.)

MARTEL, Réginald, "Et de brefs comptes rendus ", La Presse, 28 juin 1980, p. C-3.

ROYER, Jean, "La recherche du pays premier ", Le Devoir, 13 septembre 1980, p. 26.

\section{III.3.4. En état d'urgence}

BEAUSOLEIL, Claude, "Sur En état d'urgence de Denise Desautels", La nouvelle barre du jour, $\mathrm{n}^{\circ} 129$, septembre 1983, p. 99-100.

\section{III.3.5. L'écran}

BEAULIEU, Michel, "Une poésie d'une rare richesse ", Le Guide de Montréal-Nord, 29 juin 1983, p. 41-42.

BEAUSOLEIL, Claude, "Denise Desautels et la mémoire des mots", Le Devoir, 18 juin 1983 , p. 20. 
BOUCHARD, Christian, "Poésie québécoise ", Estuaire, n²9, automne 1983, p. 72-74.

BROCHU, André, "Flamboyants et profonds", Voix et Images, vol. IX, $\mathrm{n}^{\circ} 3$, printemps 1984, p. 161-162.

CLOUTIER, Guy, "Deux moments clés", Nuit blanche, no 11, décembre 1983/janvièr 1984, p. 11.

DUPRÉ, Louise, "Où loge le Norôit? ", Spirale, no 37, octobre 1983, p. 8.

MEADWELL, Kenneth W., "Aigu et sombre ", Littérature canadienne, $\mathrm{n}^{\circ} 102$, automne 1984, p. 95-98.

\section{III.3.6. : dimanche}

ALONZO, Anne-Marie, "Septième jour ”, La Vie en rose, septembre 1985, p. 56.

\section{III.3.7. La répétition}

BOURASSA, André-G., "Tableaux et théâtralité", Lettres québécoises, printemps 1988, p. 49-50.

BROCHU, André, "Chronique ", Voix et Images, n³ 35, hiver 1987, p. 324-325.

\section{III.3.8. Écritures/Ratures}

BIRON, Normand, “Le corps s'écrit-il? ", Vie des arts, n 130, mars 1988, p. 74.

DUPRÉ, Louise, "Du geste à la voix ", Trois, vol. III, nº 2, hiver 1987-1988, p. 64-66.

SASSEVILLE, Stella, "Genèse d'un projet ", Art et métiers du livre (Paris), n 156, 1989.

\section{III.3.9. Un livre de Kafka à la main}

BONENFANT, Joseph, "Des blessures toujours béantes", Estuaire, $\mathrm{n}^{\circ} 51$, hiver 19881989, p. 74-76.

BROCHU, André, "Des rats et des livres", Voix et Images, n 39, printemps 1988, p. $507-508$.

COTNOIR, Louise, "Un livre de Kafka à la main", Arcade, n 15, février 1988, p. 80-81.

DE GONZAGUE, Louise, "Un livre de Kafka à la main", Nos livres, vol. XIX, n 3 , avril 1988 , p. 35-36.

FORTIN, Marie-Claude, "Souvenirs au monde", Voir, vol. II, $\mathrm{n}^{\circ} 1$, semaine du 26 novembre au 2 décembre 1987 , p. 8.

GAUDET, Gérald, "Toucher la petite fille „, Le Devoir, 23 avril 1988, p. D-3.

HAECK, Philippe, "Penser la mort", Littérature canadienne, $\mathrm{n}^{\circ} 120$, printemps 1989 , p. 205.

HOGUE, Jacqueline, "Le moindre frémissement ", Trois, vol. V, $\mathrm{n}^{\text {os }} 1-2$, automne 1989 , p. 245.

MISTRAL, Christian, "Anecdotes, Kafka et poésie mariale ", Le Guide Mont-Royal, novembre 1987.

TOUPIN, Gilles, "Poésie d'ici „, La Presse, samedi 3 septembre 1988, p. K-2.

\section{III.3.10. Le signe discret}

PITTIER, Jacques-Michel, "Quand l'amour s'en va ", Le Matin (Lausanne), 22 mai 1988. 


\section{III.3.11. Mais la menace est une belle extravagance}

BROCHU, André, "Continuer et espérer ", Voix et Images, n 45, printemps 1990, p. 464465.

CHAMBERLAND, Roger, "Nouveautés/Poésie ", Québec français, printemps 1990, p. 18.

COATES, Carrol F., "Reviews", French Review, vol. XIIV, no 5, avril 1991, p. 871-872.

CORRIVEAU, Hugues, "La poésie est une extravagance ", Lettres québécoises, $\mathrm{n}^{\circ} 58$, été 1990, p. 35-36.

FORTIN, Marie-Claude, "Trêve de mots", Voir, semaine du 30 novembre au 6 décembre 1989, p. 24.

GAUDET, Gérald, "Mais la menace est une belle extravagance", La Poésie au Québec (revue critique 1989), Trois-Rivières, Les Écrits des Forges/Cégep JolietteDe-Lanaudière, 1990, p. 47.

GIRARD, André, "Mais la menace est une belle extravagance", Nuit blancbe, $\mathrm{n}^{\circ} 41$, octobre-novembre 1990, p. 16.

LATENDRESSE, Claude, "Lès paysages du cœur", Estuaire, n $^{\circ}$ 57, septembre 1990, p. 69-73.

LEROUX, Pierre, "Denise Desautels: la vie complexe ", Le Journal de Montréal, 24 novembre 1990 , p. We-9.

ROYER, Jean, "Poésie ", Le Devoir, samedi 19 mai 1990, p. D-3.

\section{III.3.12. Leçons de Venise}

CHAMBERLAND, Roger, "Leçons de Venise ", Québec français, n 81, printemps 1991, p. 20.

CORRIVEAU, Hugues, *Il n'y a pas de frontière, et tout est si étrange•, Trois, vol. VII, $\mathrm{n}^{\circ} 1$, automne 1991/hiver 1992, p. 108-111.

COTNOIR, Louise, "Poésie ", Arcade, no 21, printemps 1991, p. 125-126.

DORION, Hélène, "Poésie via Québec", Fond(s) de tiroir (Nantes), no 11 , décembre 1990.

FELX, Jocelyne, “ Remuer ciel et terre „, Lettres québécoises, nº 64, hiver 1991-1992, p. 36-37.

GIRARD, Marie-Claire, "Réflexions sur Venise ", Le Droit, 2 février 1991, p. A-11.

ISSENHUTH, Jean-Pierre, "Chant et désenchantement", Le Devoir, samedi 19 octobre 1991 , p. D-7.

LATENDRESSE, Claude, "La Circulation du désir", La Poésie au Québec (revue critique 1990), Trois-Rivières, Les Écrits des Forges/Cégep Joliette-De-Lanaudière, 1991, p. 59-60.

MALENFANT, Paul Chanel, "Chronique de poésie", Voix et Images, $\mathrm{n}^{\circ} 48$, printemps 1991, p. 20.

MOORHEAD, Andrea, "Reviews ", French Review, vol. XLVI, n 4, mars 1993, p. 683-684.

THIBODEAU, Serge Patrice, "Hors d'œuvres", Voir, semaine du 21 au 27 février 1991, p. 20. 


\section{III.3.13. Le saut de l'ange}

BONIN, Linda, "La trajectoire des signes ", Trois, vol. IX, n² 2, hiver 1994, p. 7-14.

BONIN, Linda, "Des voix intermédiaires", Trois, vol. X, n 3, 1995, p. 237-240.

BOURASSA, Lucie, "L'intimisme généralisé ", Le Devoir, 24 décembre 1993, p. C-13.

CLOUTIER, Guy, "Le saut de l'ange ", Le Soleil, 15 février 1994.

CRON, Marie-Michèle, "Denise Desautels et Le saut de l'ange", Le Devoir, 17 décembre 1992 , p. B-4.

GAUDET, Gérald, „Denise Desautels reçoit les prix de poésie... *, Estuaire, n 68 , printemps 1993, liminaire.

GIRARD, Marie-Claire, "Le saut de l'ange est primé ", Le Devoir, 16 février 1993, p. B-8.

LAMARRE, André, "Denise Desautels et l'écrit d'art ", Spirale, été 1993, p. 9.

LAURIN, Danielle, "Le saut de l'angen, Voir, semaine du 18 au 24 février 1993, p. 21.

MALENFANT, Paul Chanel, "De l'art de voir", Voix et Images, $n^{\circ} 54$, printemps 1993 , p. 622-624.

MONTESSUIT, Carmen, "Denise Desautels: la richesse de la poésie ", Le Journal de Montréal, 24 décembre 1993, p. We-10.

ROCHETTE-CASTEL, Céline, "Au nom de la fille, du père et de l'ange ", Gare maritime (Revue de la Maison de poésie de Nantes et Région), nº 2, mars 1995, p. 25.

TOUPIN, Gilles, "La gravité de Denise Desautels, prix de poésie du gouverneur général ", La Presse, 28 novembre 1993, p. B-5.

\section{III.3.14. Lettres à Cassandre}

BEAULIEU, Blanche, "Lettres à Cassandre", Nuit blanche, $\mathrm{n}^{\circ}$ 59, printemps 1995, p. 4041.

CORRIVEAU, Hugues, "Correspondances", Trois, vol. X, nº 3, 1995, p. 217-221.

DE GONZAGUE-PELLETIER, Louise, «Recensions", Envol, vol. III, n ${ }^{\text {os }} 1-2,1995$, p. $72-$ 73.

NEUVILLE, Laure, "C'est la nuit qu'il est beau de croire à la lumière", Homo sapiens, $n^{\circ} 23$, septembre 1995.

ROBITAILLE, Martin, “L'épistolaire en mal de lettre ", Spirale, septembre-octobre 1995, p. 31 .

\section{III.3.15. Cimetières : la rage muette}

CANTIN, David, "Théâtre du deuil ", Le Devoir, 17 février 1996, p. D-4.

CORRIVEAU, Hugues, "Il pleut sur les cimetières et sur les villes *, Lettres québécoises, $\mathrm{n}^{\circ} 83$, automne 1996 , p. 35-36.

COUËLLE, Jennifer, "Des cadavres exquis de la photographie", Le Devoir, 22 février 1996, p. B-6.

FILTEAU, Claude, *L'énigme du "Il y a ", Voix et Images, n 63, printemps 1996, p. $602-603$.

MALENFANT, Paul Chanel, "Chronique poésie ", Estuaire, n 86, hiver 1997, p. 81-92. 
MONTESSUIT, Carmen, "Les poèmes de Denise Desautels pour donner tout son mordant à "La rage muette" ", Le Journal de Montréal, 17 mars 1996, p. 54.

\section{III.3.16. "Ma joie ", crie-t-elle}

CANTIN, David, “Écrire pour voir ", Le Devoir, $1^{\mathrm{er}}$ février 1997, p. D-12.

CORRIVEAU, Hugues, "Émotion simple, simple émotion ", Lettres québécoises, $\mathrm{n}^{\circ} 86$, été 1997, p. 38-39.

JOUBERT, Lucie, "La langue de l'ange ", Estuaire, $\mathrm{n}^{\circ}$ 92, printemps 1998, p. 99-100.

LACELLE, Andrée, “Recensions ", Envol, vol. V, n 3, 1997, p. 57-58.

\section{III.3.17. Ce fauve, le Bonheur}

ANONYME, "Vitrine du livre ", Le Canada français, 4 novembre 1998, B-11.

BLONDEAU, Dominique, "Je, d'elles ", Arcade, no 45, 1999, p. 93-94.

BONIN, Linda, "Parcelles d'éternité „, Trois, vol. XV, $\mathrm{n}^{\text {os }}$ 1-2-3, 1999, p. 201-204.

BORDELEAU, Francine, "Lectures ", La Gazette des femmes, vol. XX, n 5 , janvier-février 1999, p. 12.

CHAMBERLAND, Roger, "Ce fauve, le Bonbeur", Québec français, hiver 1999, n 112, p. 23.

CHARTRAND, Robert, "Dans les griffes du bonheur", Le Devoir, 26 septembre 1998, p. D-3.

COMTOIS, Michèle, "Ce fauve, le Bonbeur", Zone, décembre 1998, p. 15.

FERLAND, Léon-Gérald, "La vie fauve, et rien d'autre ", L'Express d'Outremont, 25 septembre 1998, p. 11.

LABRECQUE, Marie, "L'enfance de l'art ", Voir, semaine du 15 au 21 octobre 1998, p. 57.

MAILHOT, Laurent, "L'enfance transfigurée", Le Couac, octobre 1998, p. 8.

MARTEL, Réginald, “La fillette et le disparu *, La Presse, 4 octobre 1998, p. B-4.

PARÉ, Yvon, "Des "âmes voyageuses" aux expériences excrémentielles ", Lettres québécoises, $\mathrm{n}^{\circ} 96$, hiver 1999 , p. 30-31.

POIRIER, Maryse, "La mort aux trousses ", Spirale, $\mathrm{n}^{\circ}$ 166, mai-juin 1999, p. 16.

SAVARD, Marie-Élaine, "À livre ouvert", Art Le Sabord, n 53, 1999, p. 52.

\section{III.3.18. Direction de dossiers de revues}

DAIGLE, Carole, "Le fantasme du livre" (sur "Installations/Fictions", sous la direction de Denise Desautels et Gilles Daigneault), Le Devoir, le 13 décembre 1986, p. D-11.

DANDURAND, Anne, "Installations/Fictions - (sur "Installations/Fictions ", sous la direction de Denise Desautels et Gilles Daigneault), La Vie en rose, février 1987, p. 49.

LEPAGE, Jocelyne, "Heureusement qu'il y a Pellan!" (sur "Installations/Fictions ", sous la direction de Denise Desautels et Gilles Daigneault), La Presse, 13 décembre 1986. 
PAYANT, René, "Installations/Fictions" (sur "Installations/Fictions", sous la direction de Denise Desautels et Gilles Daigneault), Spirale, février 1987, p. 7.

RÉGIMBALD, Diane, "Livraison d'avril " (sur "Femmes de lettres", sous la direction de Denise Desautels, La nouvelle barre du jour, avril 1989, $\left.\mathrm{n}^{\mathrm{os}} 218-219\right)$, Trois, vol. V, $\mathrm{n}^{\text {os }} 1-2$, automne 1989 , p. 260-261.

\section{III.3.19. Document audio}

CHAMBERL.AND, Roger, "Alternances", Québec français, nº 92, 1994, p. 12. 